\title{
Method of application three-dimensional billiard systems in passive heating systems
}

\author{
E. Yu. Rakhimov \\ Physical-Technical Institute, SPA “Physics-Sun”, Uzbek Academy of Sciences, Tashkent, Uzbekistan \\ rey_86@mail.ru
}

PACS 05.20, 05.70

DOI 10.17586/2220-8054-2018-9-3-319-322

\begin{abstract}
In this article, the method of determining ray absorption ability by premises with insolation passive heating system by numerical experiments was studied. A heated premise (HP), having a translucent enclosure (TE), walls covered with a special smooth enclosure, was chosen as the object of study. The dynamics of the solar rays were studied as a billiard system and the reflection equations are given. Verification of result reliability was performed.
\end{abstract}

Keywords: ray absorption ability, insolation passive heating systems, 3D billiard systems.

Received: 14 March 2018

Revised: 29 March 2018

\section{Introduction}

Many systems of differential equations cannot be solved by numerous analytical calculations, if they can be solved, then with assumptions, constraints and waste of time. If they are solved with the help of numerical methods, then concrete new results are obtained. In this way, a physical process is studied that is difficult to study, with the help of mathematical modeling. The same way, one can study the dynamics of light, laser beams [1], radio and sound waves in a specific environment [2-4], and also thermal effects at heating systems [5,6].

It is easy to study the processes which occur in the modeling of light-related phenomena (to the law of geometric optics) as a billiard system (small particle movement) [4,7-11]. In this case, the solar rays propagate along straight lines and can be investigated in the system of two or three-dimensional coordinates, in the processes which occur colliding with a barrier, its energy is conserved or not (i.e. its intensity decreases without changing its velocity).

In this paper, a method of mathematical modeling is considered to determine ray absorption ability capacity by premises with insolation passive heating system.

\section{The numerical experimental part}

In order to solve this problem, a three-dimensional billiard system was used, and the model of the HP with a TE (window with widths $b_{t e}$ and heights $h_{t e}$ ) was chosen as the object of the study (Fig. 1).

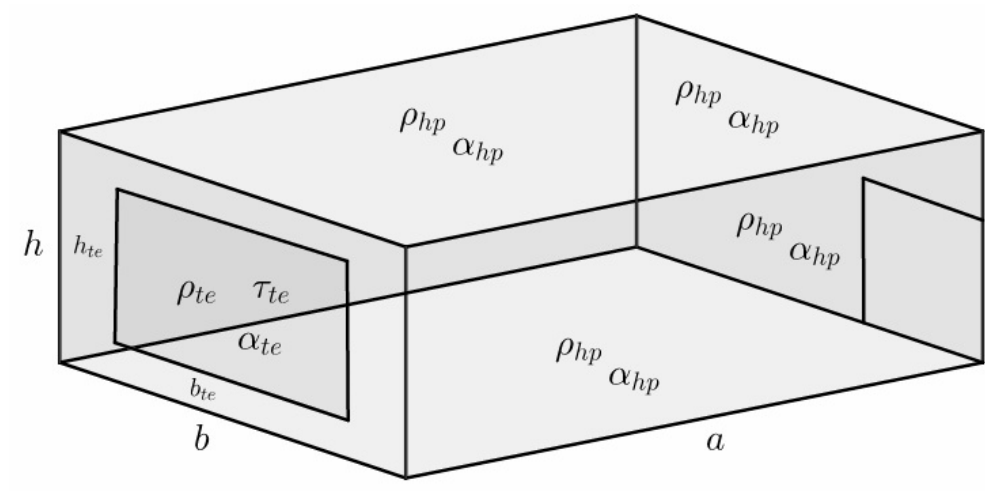

FIG. 1. Schematic diagram of the model of the HP

The following notations and conditions were introduced to simplify the modeling: 
- The reflection, absorption and transmission coefficients of solar radiation (SR) of the TE HP are equal $\rho_{t e}$, $\alpha_{t e}$ and $\tau_{t e}$, respectively;

- The walls of HP, the ceiling, the floor and the door are covered with smooth material, and solar rays falling on them obey the laws of linear geometric optics;

- The reflection and absorption coefficients of SR of a smooth material are equal to $\rho_{h p}$ and $\alpha_{h p}$, respectively;

- For direct solar radiation the reflection, absorption and transmission coefficients depend on the incidence angle of the radiation.

The part of the absorbing energy of the flux of the SR $\left(Q_{h p}^{a b s}\right)$ inside the HP that comes through the surface of the $\mathrm{TE} \mathrm{HP}$ is determined by the following equation:

$$
Q_{h p}^{a b s}=\tau_{t e} \cdot \alpha_{h p} \cdot Q_{t e}^{f a l l},
$$

where $Q_{t e}^{\text {fall }}$ - part of the falling energy of the flux of the direct SR falling on the surface of the TE HP.

If calculated by the traditional method of multiplication, $\tau_{t e} \cdot \alpha_{h p}$ from expression (1) shows that what part of the energy of the flux of the SR falling on the surface of the TE is absorbed inside the HP and is equal to:

$$
\tau_{t e} \alpha_{h p}=\frac{Q_{h p}^{a b s}}{Q_{t e}^{f a l l}} .
$$

According to the law of geometrical optics, part of the falling energy of the flux of direct SR falling on the surface of the TE HP is reflected from the surface by a factor equal to the multiplication of the $\rho_{t e} \cdot Q_{t e}^{f a l l}$ and a part is equal to the multiplication $\alpha_{t e} \cdot Q_{t e}^{\text {fall }}$ is absorbed inside the TE HP itself. Thus, the values of the part of the falling incident energy flux of the direct SR $\left(Q_{h p}^{f a l l}\right)$ to the HP, which coming through the surface of the TE $\mathrm{HP}$, is defined as following equation:

$$
Q_{h p}^{f a l l}=\tau_{t e} \cdot Q_{t e}^{\text {fall }} .
$$

During the motion of the solar rays in the HP, many times falling (collide) and reflecting on the surface of the HP and also the reverse side surfaces of the TE HP. Each time solar rays falling on the wall of the HP, part of the energy of the flux of the SR is absorbed equal to $Q_{h p_{i}}^{a b s}=\alpha_{h p} \cdot Q_{h p_{i}}^{\text {fall }}$ (where $i$ is the number of falls and reflects) and part of the energy of the flux of the SR is reflected equal to $Q_{h p_{i}}^{r e f l}=\rho_{h p} \cdot Q_{h p_{i}}^{f a l l}$, which is equal to the new value of the part of the energy of the flux of the SR for the next falling on the surface, that is, $Q_{h p_{i+1}}^{f a l l}=Q_{h p_{i}}^{r e f l}$. Also, every time when solar rays collide with the reverse side surfaces of the TE HP, part of the energy of the flux of the SR equal to $Q_{h p_{i}}^{r e f l}=\rho_{t e} \cdot Q_{h p_{i}}^{f a l l}$ is reflected on the inside of the HP and it becomes a new value for the next collision, that is, $Q_{h p_{i+1}}^{\text {fall }}=Q_{h p_{i}}^{r e f l}$.

The trajectories of solar rays as the dynamics of particle motion in the billiard system are determined.

In this case, the boundary conditions for the initial point of particle motion can be written in the plane $Y O Z$ (that is, the initial point of the particle to the area of the billiard system a located in the surface of the TE):

$$
y_{0}(z)=\left\{\begin{array}{l}
\left(b+b_{t e}\right) / 2, \\
z_{0}(y)=\left\{\begin{array}{l}
\text { if } \quad\left(h-h_{t e}\right) / 2 \leq z \leq\left(h+h_{t e}\right) / 2 ; \\
\left(h+h_{t e}\right) / 2, \\
\left(h-h_{t e}\right) / 2,
\end{array} \quad \text { if } \quad\left(b-b_{t e}\right) / 2 \leq y \leq\left(b+b_{t e}\right) / 2 .\right.
\end{array}\right.
$$

The boundary conditions of the motion of a particle in HP is in the region of $X Y Z$ and is expressed as:

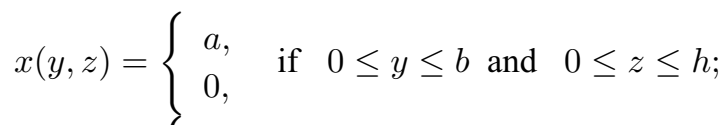

$$
\begin{aligned}
& y(x, z)=\left\{\begin{array}{l}
b, \quad \text { if } \quad 0 \leq x \leq a \text { and } \quad 0 \leq z \leq h ; \\
0, \quad ;
\end{array}\right.
\end{aligned}
$$

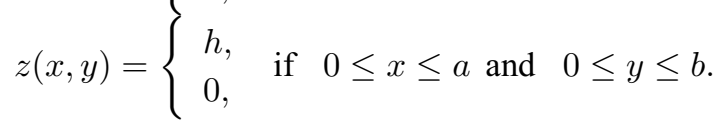

Let the particle begin its motion with a certain velocity $\vartheta_{0}$ from the surface of the TE HP, that is, from the point $\left(x_{0}, y_{0}, z_{0}\right) \in X Y Z$. Then the projections of the velocity $\vartheta_{x}, \vartheta_{y}, \vartheta_{z}$ are defined as follows:

$$
\vartheta_{x}=\vartheta_{0} \cdot \cos \beta \cdot \sin \phi ; \quad \vartheta_{y}=\vartheta_{0} \cdot \sin \beta \cdot \sin \phi ; \quad \vartheta_{z}=\vartheta_{0} \cdot \sin \phi,
$$


where $\beta$ and $\phi$ are the angles that form between the velocity vector $\vartheta_{0}$ and the unit vectors $e_{x}(i)$ and $e_{z}(i)$. Then the particle coordinates after the time $t$ can be determined by the following mappings:

$$
\begin{aligned}
& \theta_{1}=\left\{\begin{array}{ll}
\left(b-y_{i}\right) / \vartheta_{y}, & \text { if } \vartheta_{y} \geq 0 ; \\
y_{i} / \vartheta_{y}, & \text { if } \vartheta_{y}<0 ;
\end{array} \quad \theta_{2}= \begin{cases}\left(a-x_{i}\right) / \vartheta_{x}, & \text { if } \vartheta_{x} \geq 0 \\
x_{i} / \vartheta_{x}, & \text { if } \vartheta_{x}<0\end{cases} \right. \\
& \theta_{3}=\left\{\begin{array}{ll}
\left(h-z_{i}\right) / \vartheta_{z}, & \text { if } \vartheta_{z} \geq 0 ; \\
z_{i} / \vartheta_{z}, & \text { if } \vartheta_{z}<0 ;
\end{array} \quad t=\min \left(\theta_{1}, \theta_{2}, \theta_{3}\right)\right. \\
& x_{i+1}=x_{i}+\vartheta_{x} \cdot t ; \quad y_{i+1}=y_{i}+\vartheta_{y} \cdot t ; \quad z_{i+1}=z_{i}+\vartheta_{z} \cdot t .
\end{aligned}
$$

If one constructs a graphical representation of the mappings (7), obtained Fig. 2 shows the trajectories of particle motion on the planes $X O Y$ and $Y O Z$, and also at the space $X Y Z$.
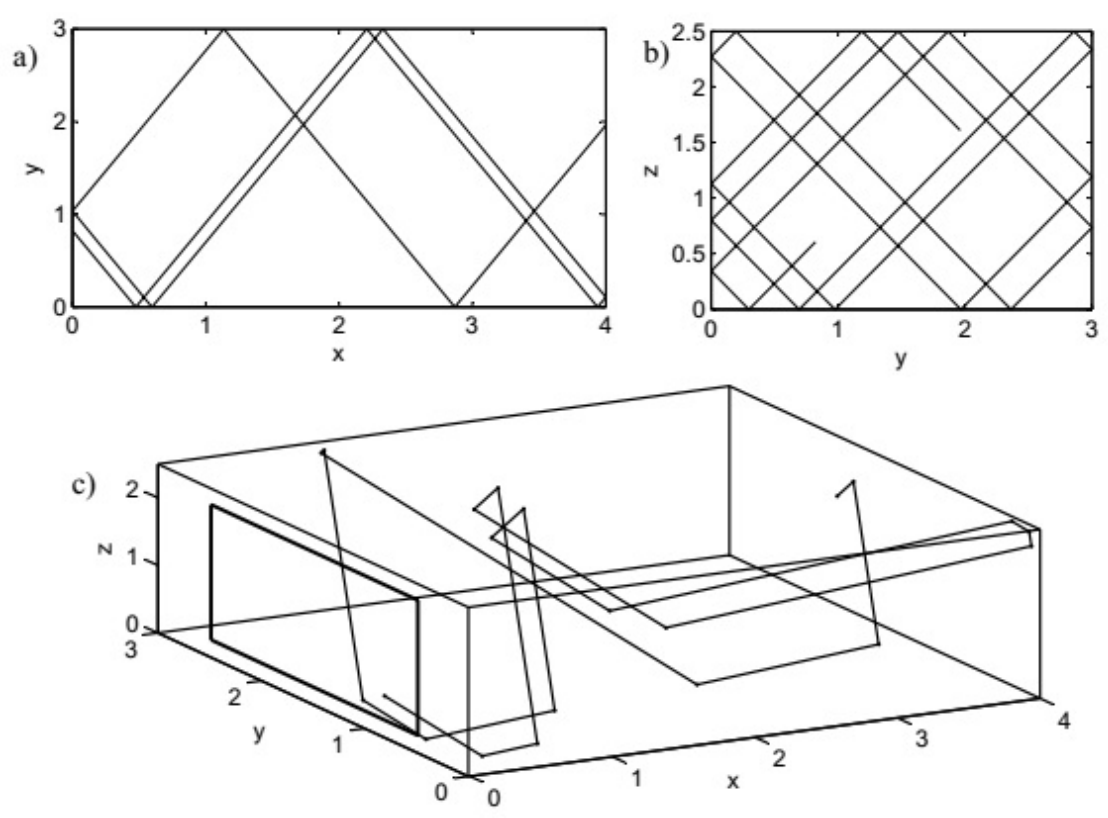

FIG. 2. Trajectory of particles in the chosen model HP: (a) at the plane $X O Y$; (b) at the plane $Y O Z$; (c) at the space $X Y Z$

Considering the optical properties of the collision of a particle with surfaces, we obtain the following result:

- when colliding with the wall surface of the HP:

$$
Q_{h p_{i+1}}^{a b s}=Q_{h p_{i}}^{a b s}+\alpha_{h p} \cdot Q_{h p_{i}}^{\text {fall }} ; \quad Q_{h p_{i+1}}^{\text {refl }}=\rho_{h p} \cdot Q_{h p_{i}}^{\text {fall }} ; \quad Q_{h p_{i+1}}^{\text {fall }}=Q_{h p_{i+1}}^{\text {refl }} ;
$$

- when colliding with the surface of the TE HP:

$$
Q_{h p_{i+1}}^{r e f l}=\rho_{t e} \cdot Q_{h p_{i}}^{\text {fall }} ; \quad Q_{h p_{i+1}}^{\text {fall }}=Q_{h p_{i+1}}^{\text {refl }} .
$$

Considering the above, we carry out the following concrete numerical experiment and for the approximation of the actual process, propagation of diffuse SR is considered. In connection with the fact that multiple internal reflection of radiation between the walls of the room is diffuse, in calculations it is used by the concept of "equivalent incidence angle of diffuse SR" which, according to [12], is taken to be $60^{\circ}$. Let the ratio of the size of the side of the HP equal to $a: b: h=3: 4: 2.5$, as well as the coefficient of absorption and reflection of the smooth material is equal to $\rho_{h p}=0.55$ and $\alpha_{h p}=0.45$. Dimensions of the TE HP with the ratio $b_{t e}: h_{h p}=2: 2$, and its optical parameters are equal to $\rho_{t e}=0.29, \alpha_{t e}=0.11$ and $\tau_{t e}=0.6$ [12]. During the numerical experiment, the optical process of $10^{4}$ times of collision and reflection of $5 \cdot 10^{3}$ particles is considered.

The results show that the ray absorption ability capacity by premises with insolation passive heating system is equal to $Q_{h p}^{a b s} / Q_{t e}^{\text {fall }}=0.608$. This value is more than 2.22 times the classical value $\left(\tau_{t e} \cdot \alpha_{h p}=0.27\right)$, determined from the expressions (1). The results obtained as a result of numerical experiments are consistent with the results obtained using the analytical method [13]. 


\section{Conclusion}

The results show that such experiments can be used to aid the design of solar devices, at the design and alignment of solar ovens, as well as focusing systems by using parabolocylindric mirrors and designing a lightreceiving system of solar tower type.

\section{References}

[1] Creagh S.C. Directional emission from weakly eccentric resonators. Phys Rev Lett., 2007, 98(15), P. 153901.

[2] Dembowski C., Gräf H-D., Heine A, Hesse T., Rehfeld H., Richter A. First experimental test of a trace formula for billiard systems showing mixed dynamics. Phys Rev Lett., 2001, 86(15), P. 3284-7.

[3] Abdullaev S.S. and Zaslavskii G.M. Classical nonlinear dynamics and chaos of rays in problems of wave propagation in inhomogeneous media. Sov. Phys. Usp., 1991, 34(8), P. 645.

[4] Loskutov A.Yu., Ryabov A.B., Akinshin L.G. Mechanism of Fermi acceleration in dispersing billiards with time-dependent boundaries. Journal of Experimental and Theoretical Physics, 1999, 89(2), P. 966-974.

[5] Eckmann J.P., Mejı́-Monasterio C. Thermal Rectification in Billiardlike Systems. Phys Rev Lett., $2006,97(9)$, P. 094301.

[6] Lee K.Y., Chi D.Y., Kim S.H., Seo K.W., and Yoon J. Passive residual heat removal system of an open-pool type research reactor using gravity core cooling tank, in Proceedings of the European Research Reactor Conference (RRFM '14), Ljubljana, Slovenia, March-April, 2014, P. 593-599.

[7] Weiss D., Roukes M.L. and et al. Electron pinball and commensurate orbits in a periodic array of scatterers. Phys. Rev. Lett., 1991, 66, P. 2790.

[8] Markarian R., Kamphorst S.O., de Carvalho S.P. Chaotic Properties of the Elliptical Stadium. Commun. Math. Phys. 1996, 174, P. 661-679.

[9] Berry M.V. Regularity and chaos in classical mechanics, illustrated by three deformations of a circular billiard. Eur. J. Phys. 1981, 2, P. 91-102.

[10] Edson D.L., Carl P.D. Recurrence of particles in static and time varying oval billiards. Physics Letters A, 2012,376 (20), P. 1669-1674.

[11] Akhmadjanov T., Rakhimov E. and Otajanov D. Particle dynamics in corrugated rectangular billiard. Nanosystems: Phys., Chem., Math., 2015, 6(2), P. 262-267.

[12] Duffie J.A., Beckman W.A. Solar Engineer of Thermal Processes. Fourth Solution. Hoboven New Jersey: John Wiley Sons. Inc., 2013, $910 \mathrm{p}$.

[13] Avezov R.R., Avezova N.R. and et al. Ray absorption ability capacity by premises with insolation passive heating system. Alternative Energy and Ecology, 2017, 07-09, P. 034-042. 